\title{
CORRECCION DE RUIDO TEMPORAL Y ESPACIAL ORIENTADO A LA MAXIMIZACIÓN DE CONTRASTE Y COMPENSACIÓN DE ILUMINACIÓN EN IMÁGENES INFRARROJAS
}

\section{TEMPORAL AND SPATIAL NOISE CORRECTION ORIENTED TO THE MAXIMIZATION OF CONTRAST AND COMPENSATION OF ILUMUNATION IN INFRARED IMAGES}

\author{
Alejandro Jaramillo I. ${ }^{1}$, José Bestier Padilla B. ${ }^{2}$, Ramiro Arango. ${ }^{3}$ \\ Programa de Tecnología Electrónica de la Universidad del Quindío-Colombia. Correo electrónico ajaramillo12@yahoo.com, \\ jbpadilla@uniquindio.edu.co,ramy@uniquindio.edu.co \\ Recibido: Enero 27 de 2011 \\ Aceptado: Noviembre 12 de 2011 \\ Correspondencia: Programa de Tecnología Electrónica, Universidad del Quindío. Carrera 15 calle 12 norte Armenia Quindío -Colombia.
}

\section{RESUMEN}

Las imágenes infrarrojas presentan defectos asociados al hardware, como son: píxeles defectuosos, ruido temporal, ruido espacial, vignetting entre otros; e inherentes al medio como cambios de iluminación, corrientes de convección y conducción. En este trabajo se hace uso de metodologías enfocadas en la corrección de no uniformidades como lo es el algoritmo de las estadisticas constantes (CS) y rango constante (CR), así mismo se utiliza la morfología matemática y mapeos de contraste (MC) con el objetivo de maximizar el contraste y compensar los cambios de iluminación de cada uno de los frames infrarrojos.

Las métricas utilizadas para evaluar el desempeño de las técnicas corresponden al RMSE (error cuadrático medio) y al índice de merito Rho el cual es un estimativo de la cantidad de ruido eliminado, los resultados obtenidos con la utilización de las técnicas de corrección de no uniformidades presentan un índice de merito Rho y un RMSE bajo en comparación a los valores de estas métricas para frames contaminados, siendo estos cercanos a valores de las métricas obtenidos para un frame sin ruido espacial, aun, mejores resultados se obtienen cuando se aplican los operadores morfológicos y mapeos de contraste a las secuencias de imágenes provenientes de la etapa de corrección de ruido, dado que el contraste de la imagen es mejorado adaptativamente.

PALABRAS CLAVES: Rango Constante, Estadísticas Constantes, Maximización de Contraste, Infrarrojo, Defectos del Hardware, Defectos del Medio, Índice de Merito, RMSE.

\begin{abstract}
The infrared images have defects associated with the hardware such as defective pixels, temporal noise, spatial noise, vignetting and so on. They are inherent to the environment as illumination changes, currents of convection and conduction. This work makes use of methodologies focused on the correction of non uniformities as it is the algorithm of constant statistics (CS) and constant rank (CR), likewise using mathematical morphology and mapeos of contrast (MC) with the aim of maximizing the contrast and compensate the illumination changes from each of the infrared frames.

The metrics used to evaluate the performance of the techniques correspond to RMSE (half quadratic error) and the index of merit Rho which is an approximation of the amount of noise removed, the results obtained with the use of the correction techniques of non uniformities have an index Rho and a low RMSE compared to the values of these metrics for contaminated frames, Being these frames close to the metrics values obtained for a frame without spatial noise, we can even obtain better results when we apply morphological operators and mapeos of contrast to the sequences of images that come out of the stage noise correction given that the image contrast is improved adaptively.
\end{abstract}

KEYWORDS: Constant rank, constant statistics, maximizing the contrast, infrared, hardware defects, environment defects, index of merit, RMSE. 


\section{INTRODUCCIÓN}

En los últimos años las imágenes infrarrojas han mostrado gran utilidad en áreas como las ciencias básicas, medicina, seguridad y vigilancia entre otras, permitiendo el desarrollo y elaboración de procesos que anteriormente mostraban limitantes en su ejecución. Los principales problemas al trabajar con este tipo de imágenes se presentan debido a las deficiencias asociadas al hardware, es decir características en cuanto a la electrónica y a las especificaciones técnicas con las que se construyo la cámara, encontrando problemas de píxeles parpadeantes(1), píxeles de derivación o drifting(2), ghosting(3), píxeles defectuosos(4) ruido temporal(1), ruido espacial(1) y vignetting(4); así mismo existe otro tipo de deficiencias los cuales son asociadas al medio de adquisición donde se tome la escena, siendo estas debidas a cambios de iluminación, corrientes de convección y conducción (1). Con el ánimo de disminuir o corregir los problemas anteriormente citados se han desarrollado trabajos en áreas como la morfología matemática, modelado, preprocesado y corrección de no uniformidades los cuales son utilizados en este trabajo con el propósito mejorar las características de la imagen como lo son el contraste, equilibrio en la iluminación y fidelidad (asociada al ruido).

El resto de este documento está organizado de la siguiente manera. En la sección II se presentan los antecedentes de algunas de las metodologías utilizadas para la corrección de defectos en las imágenes infrarrojas, en la sección III se presentan el método de maximización de contraste y compensación de Iluminación, en la sección IV se presenta el método del rango constate, en la sección $\mathrm{V}$ se encuentra el desarrollo conceptual del método de las estadísticas constantes, en la sección VI se describen los métodos e implementos utilizados en el desarrollo de este trabajo, en la sección VII se muestran los resultados obtenidos para la corrección de no uniformidades y maximización de contraste, en la sección VIII se exponen las conclusiones del trabajo realizado y por ultimo en la sección IX se encuentran las referencias utilizadas en este trabajo.

\section{ANTECEDENTES}

Teniendo en cuenta los factores que causan un constante deterioro en la calidad de las imágenes infrarrojas, existen diferentes metodologías que buscan corregir los defectos, así mismo maximizar el contraste de la imagen y compensar la iluminación. Estas metodologías se basan en el uso de modelos, preprocesado, morfología matemática y corrección de no uniformidades.

En las metodologías basadas en modelos, se parte de un conjunto de imágenes de entrenamiento, capturadas en condiciones de iluminación variables; en donde el modelo de lluminación Lambertiano (6) es comúnmente empleado para describir un objeto en la imagen. Dentro de esta categoría se encuentran los conos de iluminación (7) (8) y los armónicos esféricos (9).

Por su parte, en la metodología conocida como preprocesado, las imágenes son previamente transformadas usando alguna técnica con la finalidad de ser normalizadas y que aparezcan estables bajo diferentes condiciones de iluminación. Las técnicas implementadas para este propósito se basan en funciones no lineales como logaritmos o potencias, las cuales trabajan a nivel de píxel (10). Con el mismo objeto pero trabajando en el dominio de la frecuencia se encuentra el denominado filtrado homomórfico, el cual ha presentado buenos resultados trabajando sobre imágenes infrarrojas (11).

Por otra parte una de las metodologías más utilizadas corresponde a la ecualización de histograma global y local. Sin embargo, la principal desventaja de la ecualización de histograma es que las propiedades globales de la imagen no pueden ser aplicadas apropiadamente en un contexto local, lo que conduce a un desempeño pobre en cuanto a la preservación de detalles.

A continuación se presentan los fundamentos teóricos de cada una de las técnicas utilizadas para la corrección de no uniformidades, como lo es la técnica del rango constante y estadísticas constantes, de igual forma este procedimiento se efectúa para la metodología que se centra en el uso de morfología matemática y mapeos de contraste.

\section{Maximización de contraste y compensación de iluminación}

La metodología basada en morfología matemática y mapeos de contraste se fundamenta en la aplicación de la ley de Weber, transformaciones morfológicas y mapeos de contraste cuyo método fue desarrollo en (12). 
Los fundamentos matemáticos de las técnicas para la maximización de contraste y compensación de iluminación $(\mathrm{MC})$ se describen a continuación.

\section{A. Ley de Weber}

La ley de Weber corresponde a un modelo de percepción visual, el cual indica que la razón entre la magnitud del estímulo necesario para percibir un cambio en la sensación apenas notable $\Delta L$, y la iluminación de fondo $L$, es una constante $\Delta L / L=$ const Así mismo la ley de Weber también puede expresarse por medio de la ecuación logarítmica

$$
C=a_{1}+a_{2} \log L \quad L>0 .
$$

\section{B. Apertura y Cierre por Reconstrucción}

En morfología matemática, los filtros básicos son la apertura morfológica $\gamma_{\mu B}$ yelcierre morfológico $\varphi_{\mu B}$ por un elemento estructurante $B, \bar{B}$ es el conjunto transpuesto $(\bar{B}=\{-x: x \in B\})$ y $\mu$ es un parámetro de tamaño. De esta manera, la apertura y cierre están dadas respectivamente por:

$$
\begin{gathered}
\gamma_{\mu B}(f)(x)=\delta_{\mu B}\left(\varepsilon_{\mu B}(f)\right)(x) \\
\phi_{\mu B}(f)(x)=\varepsilon_{\mu B}\left(\delta_{\mu B}(f)\right)(x) .
\end{gathered}
$$

Donde la erosión y la dilatación se definen en (4) y (5) respectivamente.

$$
\begin{aligned}
& \varepsilon_{\mu B}(f)(x)=\wedge\left\{f(y): y \in \mu \bar{B}_{x}\right\} \\
& \delta_{\mu B}(f)(x)=\vee\left\{f(y): y \in \mu \bar{B}_{x}\right\}
\end{aligned}
$$

Para efectuar los respectivos mapeos de contraste en la imagen se debe hacer el uso de dos funciones o primitivas descritas en las ecuaciones (6) y (7) con base en el fondo de la imagen; es así que cada primitiva será aplicada bajo un criterio de fondo obtenido a partir de $b_{1 \mathrm{y}} b_{2}(12)$ :

$$
\begin{aligned}
& K_{b 1, b 2}(x)=K_{1} \log (f(x)+1)+b 1 \\
& K_{b 1, b 2}(x)=K_{2} \log (f(x)+1)+b 2
\end{aligned}
$$

Donde

$$
\tau=\frac{\left(b_{1}+b_{2}\right)}{2}
$$

Los valores $b_{1}$ y $b_{2}$ asociados al fondo de la imagen se obtienen a partir del análisis de los mínimos regionales; en donde la intensidad de los píxeles correspondientes al mínimo regional más grande se asociará a $b_{1}$; mientras que el valor del mínimo regional más pequeño se asocia a $b_{2}$.

Por otro lado las técnicas que se basan en la corrección de defectos del hardware que corresponden a una característica asociada a los dispositivos fotográficos se denominan metodologías basadas en corrección de no uniformidades o también conocidas como corrección de ruido espacial. En este trabajo se hace la implementación del algoritmo del rango (CR) constante y estadísticas constantes (CS) descrito por (1).

\section{MATERIALES Y MÉTODOS}

\section{A. Método del rango constante}

En esta técnica se supone una distribución estadística para la radiación infrarroja de tipo uniforme como se observa en la ecuación (9), esto debido a que no se sabe a priori la distribución que esta puede tener, sin embargo de lo que si se está seguro es que la radiación incidente se puede ubicar dentro de márgenes conocidos (1). Así, se espera que todas las radiaciones ubicadas dentro de dicho rango tengan la misma probabilidad de incidir en la matriz de detectores. La forma utilizada para encontrar dicho rango que se le atribuye a la radiación infrarroja es basada en histogramas. Esto es, se utiliza un bloque de frames capturados por la cámara, que se someten a una evaluación estadística que genera dos matrices, una con los máximos de cada píxel en ese bloque y otra con los mínimos. Luego, a cada una de las matrices anteriores se les extrae la media. Dichos valores corresponden al rango que se le asocia a la radiación infrarroja incidente. El modelo matemático para el método del rango constante es el siguiente.

$$
\begin{gathered}
X \approx \text { Uniforme }\left[X_{\min }, X_{\max }\right] \\
\bar{X}=\frac{X_{\max }+X_{\min }}{2}
\end{gathered}
$$

$$
\sigma_{X}^{2}=\frac{\left(X_{\max }+X_{\min }\right)^{2}}{12}
$$


Donde:

$\bar{X}$ :representa la media de la radiación infrarroja incidente en el plano focal infrarrojo.

$\sigma_{X}^{2}$ : representa la varianza de la radiación infrarroja incidente en el plano focal infrarrojo.

Ahora se considera el modelo del detector infrarrojo el cual se describe en la ecuación (12).

$$
Y_{i j}(n)=A_{i j} \cdot X_{i j}(n)+B_{i j}
$$

Donde

$Y_{i j}:$ Respuesta del detector del píxel $(i, j)^{\text {avo }}$

$A_{i j}$ : Ganancia del detector del píxel $(i, j)^{\text {avo }}$

$X_{i j}(n)$ : Radiación infrarroja pura que llega al detector

$(i, j)^{\text {avo }}$ en el frame $n$.

$B_{i j}$ : Offset de voltaje del detector correspondiente al píxel $(i, j)^{\text {avo }}$.

Los parámetros de ganancia y offset se pueden obtener de la forma:

$$
\begin{gathered}
A^{2}{ }_{i j}=\frac{\sigma_{Y i j}^{2}}{\sigma_{X i j}^{2}} \\
B_{i j}=\bar{Y}_{i j}-A_{i j} \cdot \bar{X}_{i j}
\end{gathered}
$$

Y finalmente la estimación de la radiación infrarroja se define como:

$$
X_{i j}=\begin{gathered}
Y_{i j}-B_{i j} \\
A_{i j}
\end{gathered}
$$

\section{B. Método de las constantes estadísticas}

Presentado por Harris et al (14), propone la asignación de una distribución estadística Normal a la radiación infrarroja, con media cero y varianza unitaria. Considera un modelo matemático del detector, pero no incluye el ruido temporal al suponer que el nivel de incidencia de dicho factor es mínimo en la salida del sistema. Además, impone que la intensidad media de todos los pixeles de la matriz de detectores debe ser idéntica y la varianza de la radiación de entrada es idéntica para cada píxel. El modelo matemático considerado es el siguiente.

$$
y(t)=a x(t)+b
$$

Donde:

$y(t)$ : es la variable medida

$a$ : es la ganancia asociada a la detección.

$x(t)$ : es la radiación infrarroja obtenida desde el mundo real.

$b$ : es el offset asociado a la detección.

Para determinar la ganancia y el offset necesarios para generar las correcciones, el algoritmo depende de las estadísticas directas del modelo, por lo que se extrae media y varianza de la ecuación (16).

$$
\begin{gathered}
m_{y}=E[y]=E[a x+b]=a \cdot m_{x}+b \\
\sigma_{y}^{2}=\operatorname{var}[y]=a^{2} \cdot \sigma_{x}^{2}
\end{gathered}
$$

Por lo que la ganancia $a$ y el offset $b$ se expresan en las ecuaciones (19) y (20), debido a que se asume $\bar{x}=0$ y $\sigma_{x}^{2}=1$.

$$
\begin{aligned}
& b=E[y]=m_{y} \\
& a=\frac{\sigma_{y}}{\sigma_{x}}
\end{aligned}
$$

Así, se determina la radiación infrarroja

$$
x=\frac{y-m_{y}}{\sigma_{y}}
$$

A continuación se describen los métodos, implementados y practicas desarrolladas en este trabajo para la corrección de no uniformidades maximización de contraste y compensación de iluminación.

\section{Construcción de la Base de Datos}

La base de datos se construyó usando secuencias de video con baja y alta variabilidad de los objetos en la escena, con el propósito de observar el comportamiento de los algoritmos ante estas condiciones, ya que para establecer la radiación infrarroja estos dependen de la varianza, por lo tanto escenas tomadas a objetos que permanecen estáticos por mucho tiempo, pueden causar indeterminaciones en la estimación de la radiación infrarroja. 
La cámara infrarroja utilizada para efectuar la adquisición de los videos corresponde a una Optitrack modelo FLEX:C120, cada secuencia tomada a las 1:30PM, 2:45PM, 3:45PM, 4:30PM y 5:45PM horas, cada una de aproximadamente 450 frames. Estas secuencias de video son tomadas a diferentes horas con el propósito de observar la incidencia que tiene la baja o alta exposición de la radiación infrarroja ante las correcciones efectuadas por los algoritmos, de igual forma a cada una de las secuencias de video adquiridos se contaminan con $10 \%$ y $35 \%$ de ruido espacial para efectuar su posterior corrección.

\section{Corrección de Ruido}

En esta etapa se procede a corregir el ruido espacial utilizando los algoritmos de las estadísticas constantes y rango constante, a estos dos algoritmos se les entrega por separado una secuencia de aproximadamente 450 frames correspondientes a cada una de las secuencias que se adquirieron a diferentes horas del día; en algunos casos se pueden presentar problemas en cuanto al uso de la memoria virtual del equipo de computo que se esté utilizando, sin embargo si no se presentan problemas con secuencias mayores a 450 frames es bueno hacerlo ya que de esta forma las correcciones efectuadas obtiene mejores rendimientos.

Posterior a la corrección efectuada con los algoritmos de rango constante y estadísticas constantes se procede a mejorar el contraste de cada uno de los frames, en la ejecución de este algoritmo se utilizo un elemento estructurante de tamaño 15 , se debe tener en cuenta que los valores del elemento estructurante entre 15-21 permiten detectar mínimos ubicados en ojos nariz y boca (12) en el caso en que este algoritmo sea utilizado como etapa previa a la detección de rostros.

\section{E. Métricas de Evaluación}

Las métricas establecidas para la evaluación de las técnicas en consideración corresponden al RMSE o error cuadrático medio el cual se utiliza para determinar una medida de distancia entre las correcciones y un patrón óptimo; así mismo el índice de merito Rho se utiliza para cuantificar la efectividad de la corrección de no uniformidades, por medio de la detección de suavidades tanto en la imagen real como en la imagen corregida, cada uno de los parámetros nombrados anteriormente se calculan con ventanas de estimación creciente.

Las expresiones utilizadas para calcular el RMSE y el Índice de merito Rho son las siguientes:

$$
\operatorname{RMSE}\left(x_{k}, x_{t p c}\right)=\sqrt{\frac{1}{N \cdot M} \cdot \sum_{t=1}^{N \cdot M}\left(x_{k}(i)-x_{t p c}(i)\right)}
$$

\section{Donde}

: es la imagen corregida a comparar.

: es el patrón con el que se compara.

$N$ : numero de filas del arreglo.

$M$ : numero de columnas del arreglo.

$$
\rho(f) \frac{\left\|h^{*} f\right\|_{l}+\left\|h^{T} * f\right\|_{l}}{\|f\|_{1}}
$$

$\rho(f)$ : Índice de mérito $R$ ho

$f$ : es la imagen a evaluar.

$h$ : es una máscara horizontal encargada de evaluar

la suavidad de la imagen. $h=(1,-1)$.

* : representa la convolución discreta.

\|. . $\|_{l}$ : representa la norma $L_{1}$ de su argumento.

\section{RESULTADOS}

A. Corrección de no uniformidades utilizando rango constante y maximización de contraste

En la Figura 1 se muestra un frame aleatorio adquirido a las 1:30PM corregido por el algoritmo de rango constante, contaminado con $10 \%$ de ruido espacial
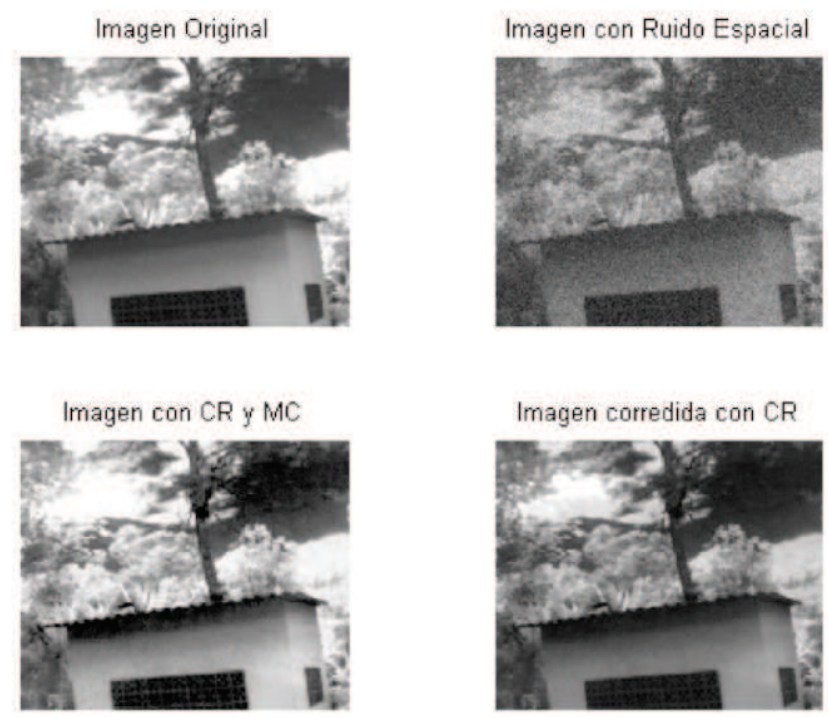

Figura 1. Corrección frame aleatorio, 1:30PM 


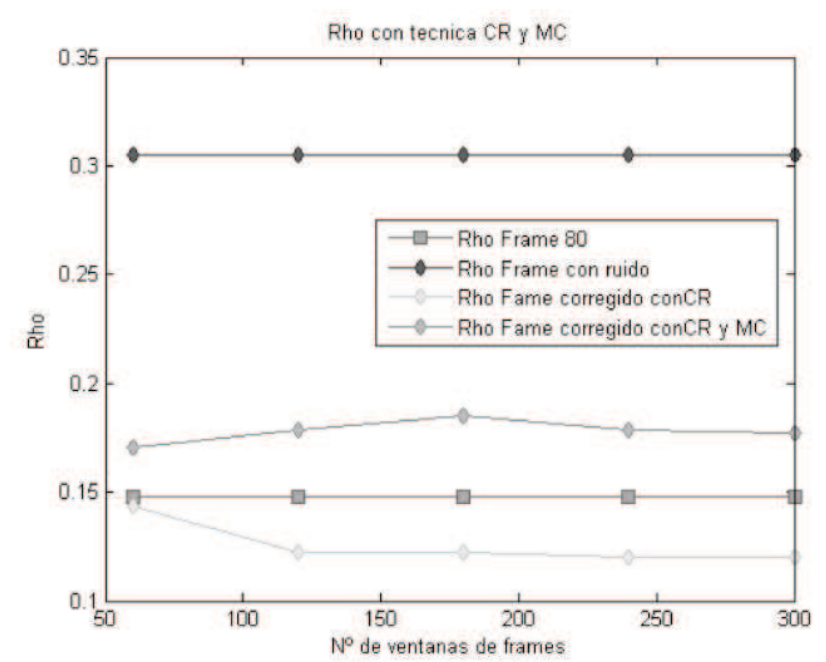

Figura 2. Coeficiente de rugosidad frame aleatorio, 1:30PM

En la Figura 1, se observa el resultado de aplicar la técnica de rango constante maximización de contraste, con el fin de corregir el ruido espacial presente en un frame aleatorio.

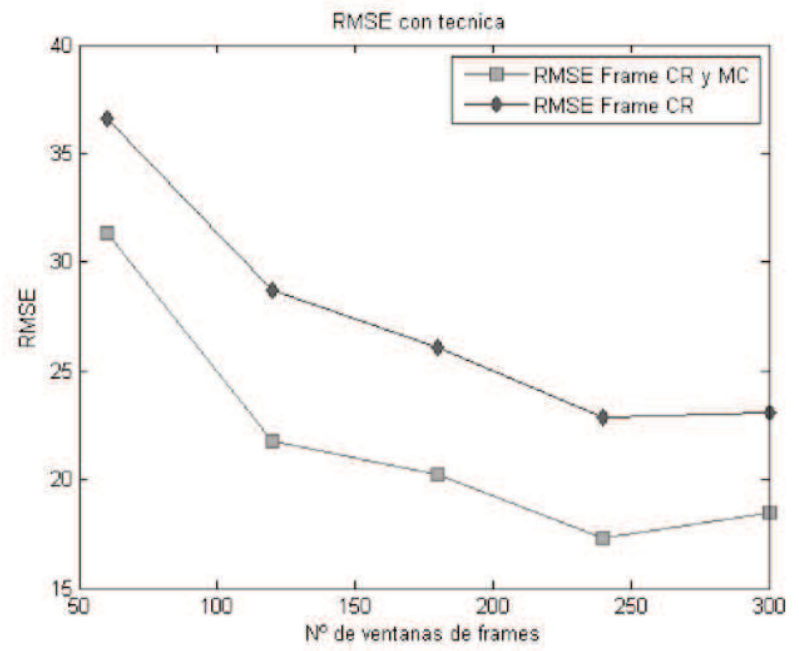

Figura 3 . Figura de merito RMSE frame aleatorio 1:30PM

Para probar el desempeño de la técnica de corrección de no uniformidades, se aplico en la imagen inicialmente la técnica de RC y luego RC+MC. Si bien el resultado de la corrección del ruido espacial muestra un buen desempeño al aplicar las dos técnicas, en las figuras 2 y 3 se observan los índices de desempeño para cada imagen corregida ya sea con RCó RC+MC.

La Figura 2 muestra que el coeficiente de rugosidad en las imágenes corregidas con CR y CR+MC es mucho más bajo que el de la imagen contaminada con ruido espacial; lo que implica que las técnicas de CR y CR+MC muestran un buen desempeño en la corrección de no uniformidades, ya que eliminan en gran parte el ruido espacial presente en las imágenes. Aunque el coeficiente de rugosidad de la imagen corregida con $\mathrm{CR}+\mathrm{MC}$ sea mayor que al aplicar la técnica de $\mathrm{CR}$, no implica que la corrección sea de bajo desempeño, ya que la técnica de maximización de contraste luego de corregir el ruido espacial, mejora el contraste en la imagen añadiendo altas frecuencias espaciales.

Otro índice de desempeño para la corrección de no uniformidades se muestra en la Figura 3, en la cual se observa que el RMSE de la imagen corregida con CR y $\mathrm{CR}+\mathrm{MC}$ disminuye, si se aumenta el número de frames en la estimación de la radiación infrarroja, en contraste con la figura 2, el RMSE de la imagen corregida con $\mathrm{RC}+\mathrm{MC}$ es menor que el de la técnica $\mathrm{CR}$, debido a que la imagen original presenta zonas donde el contraste es más marcado, por lo que al aplicar la técnica de CR+MC, la imagen corregida se beneficia de la técnica de maximización de contraste.

La Figura 4 muestra un frame aleatorio corregido por el algoritmo de rango constante, el cual ha sido contaminado con $10 \%$ de ruido espacial adquirido a la 3:30PM.
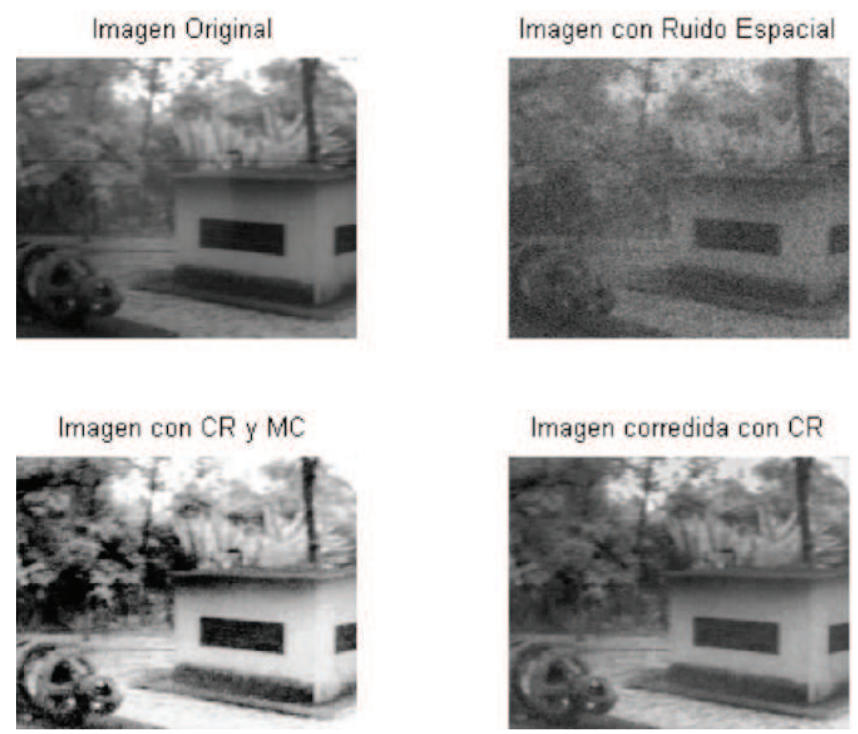

Figura 4 . Corrección frame aleatorio, 3:30PM 


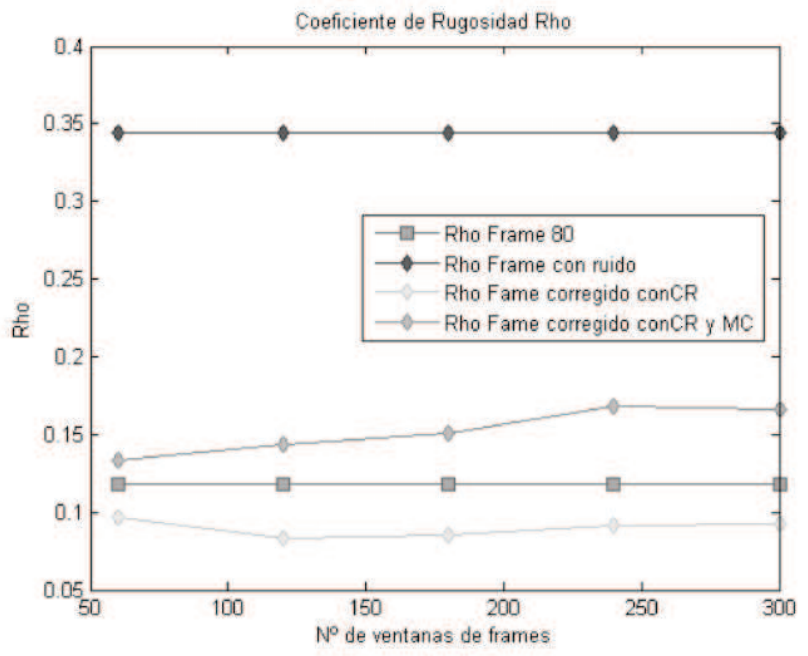

Figura 6 . Coeficiente de rugosidad frame aleatorio, 3:30PM

En la figura 4, se observa el resultado de aplicar la técnica de rango constante (CR) y maximización de contraste (MC), con el fin de corregir el ruido espacial presente en un frame aleatorio. Para probar el desempeño de la técnica de corrección de no uniformidades, se aplico en la imagen inicialmente la técnica de RC y luego RC+MC. Si bien el resultado de la corrección del ruido muestra un buen desempeño al aplicar las dos técnicas, en las Figuras 5 y 6 se observan los índices de desempeño para cada imagen corregida ya sea con RC ó RC+MC.

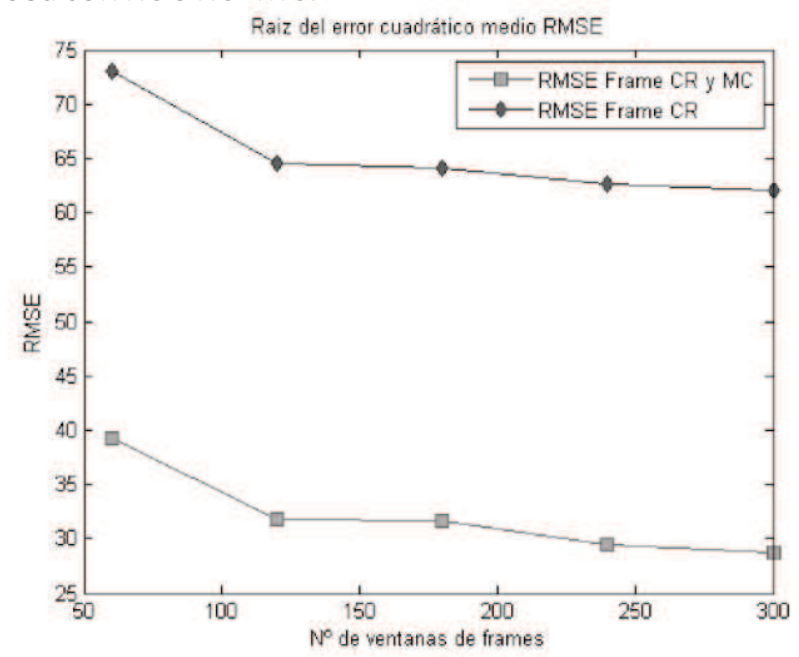

Figura . Figura de merito RMSE frame aleatorio 3:30PM

Al igual que en la Figura 2, el coeficiente de rugosidad de la Figura 5, evidencia el buen desempeño de la técnica de rango constante, ya que elimina las altas frecuencias presentes en la imagen, con lo cual se corrige el ruido espacial presente en la imagen.

En la Figura 6, se evidencia el incremento del RMSE al aplicar las técnicas de RC y RC+MC, debido a que la hora en la que se grabo la escena presentaba una iluminación menor, por lo cual la imagen corregida con RC presenta un contraste más bajo, además la estimación de la radiación se ve afectada por la baja variabilidad de los pixeles en cada frame.
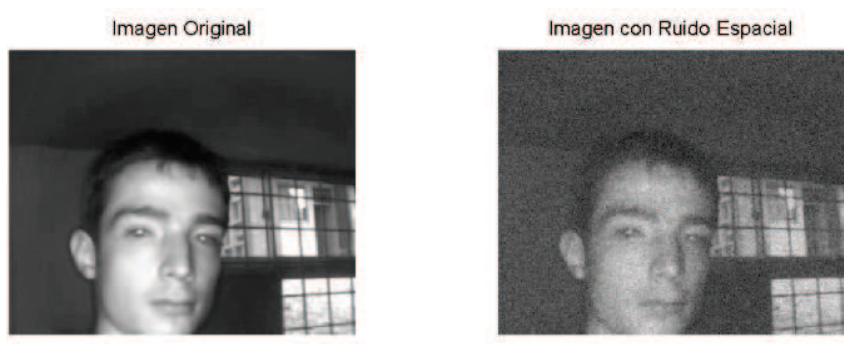

Imagen con $\mathrm{CR}$ y $\mathrm{MC}$

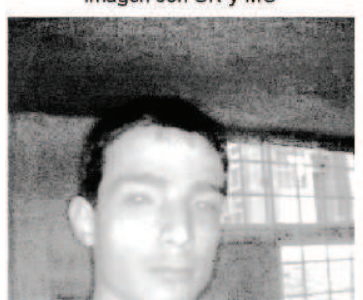

Imagen corredida con $\mathrm{CR}$

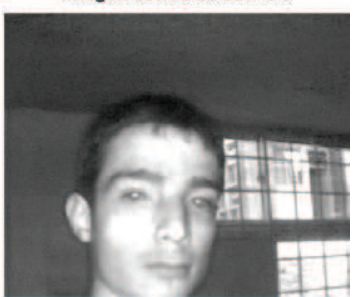

Figura 7 . Corrección frame aleatorio, 5:45PM

En un ambiente de baja iluminación como el que se muestra en la Figura 7, al aplicar las técnicas de CR y $\mathrm{CR}+\mathrm{MC}$, se nota claramente que el desempeño de la corrección de no uniformidades disminuye claramente, esto debido a que en ambientes de baja iluminación en el momento de estimar la radiación infrarroja, la variabilidad de los pixeles en cada frame será muy pequeña por lo que la radiación infrarroja tendrá una pobre estimación. 


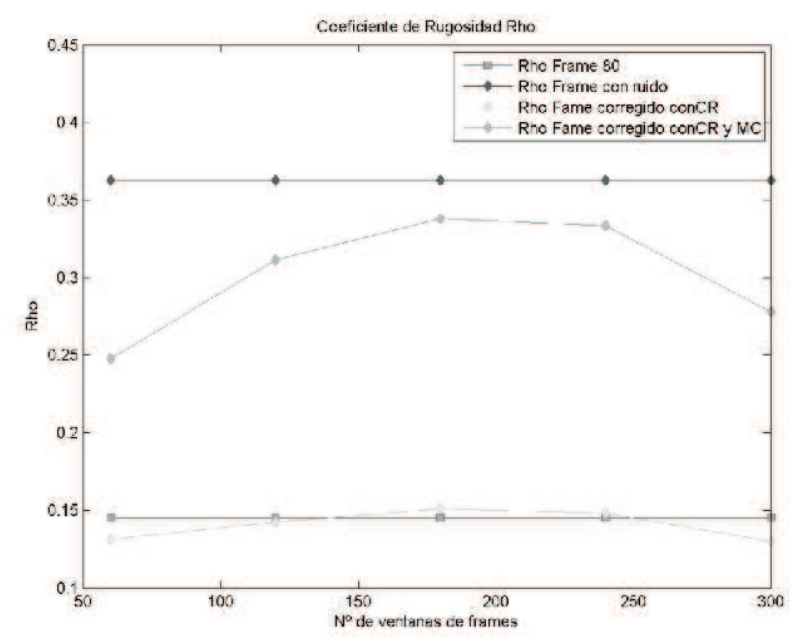

Figura 8. Coeficiente de rugosidad frame aleatorio, 5:45PM

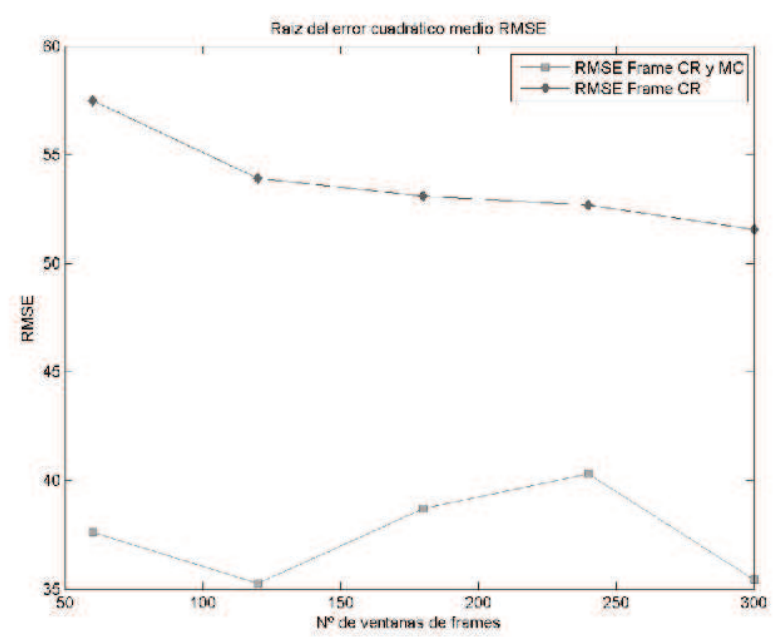

Figura 9. Figura de merito RMSE frame aleatorio 5:45PM

Este error producido por la baja iluminación se evidencia en las Figuras 8 y 9 , donde el coeficiente de rugosidad de la imagen corregida con CR+MC aumenta de manera notoria ocasionando que la imagen presente un contraste demasiado alto, además el RMSE de la imagen corregida al aplicarle las técnicas de $\mathrm{RC}$ y $\mathrm{RC}+\mathrm{MC}$ aumenta significativamente, esto debido a la técnica de $\mathrm{CR}$, la cual en ambientes de baja iluminación, presenta deficiencias en la estimación de la radiación infrarroja como se menciona anteriormente.

Adicionalmente el cambio de comportamiento del RMSE en la Figura 9 donde se observa el aumento y posteriormente un declive de esta métrica, se debe a que si se toma una ventana de estimación de 160 frames y esta secuencia posee una cantidad considerable de frames donde la iluminación de la escena es baja la estimación de la radiación infrarroja no será la más adecuada, además a este comportamiento la técnica de MC puede incrementar este valor, es importante aclarar que en posteriores ventanas la iluminación ya haya mejorado haciendo que el RMSE baje por este motivo es bueno hacer una estimación de la radiación con el mayor número de frames que se pueda.

\section{A. Corrección de no uniformidades utilizando estadísticas constantes y maximización de contraste \\ De igual forma que en la corrección de no uniformidades utilizando la técnica de rango constante y maximización de contraste, se toma un frame aleatorio el cual está contaminado con ruido espacial del $10 \%$, la corrección de este frame adquirido a la 1:30PM se muestra en la fioura 10}

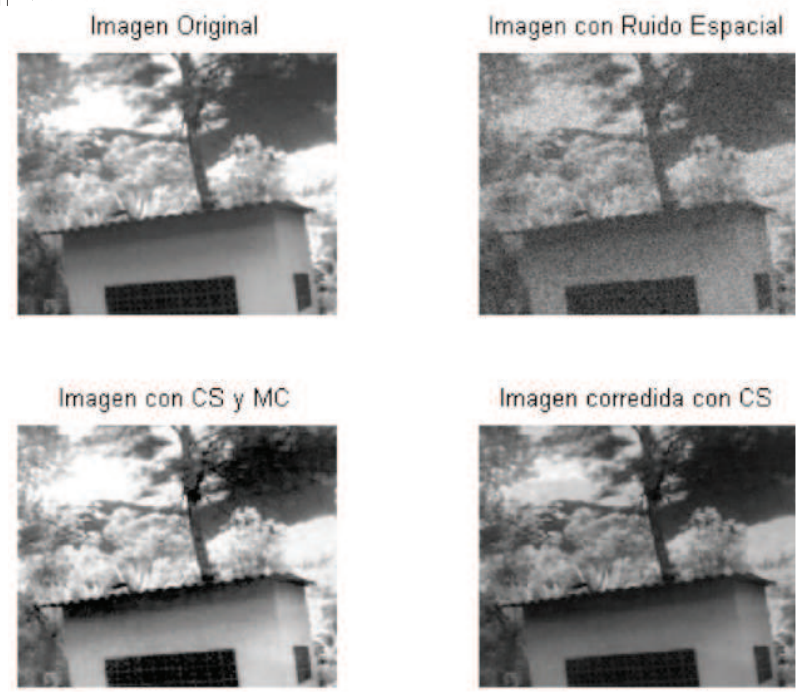

Figura 10. Corrección frame aleatorio, 1:30PM

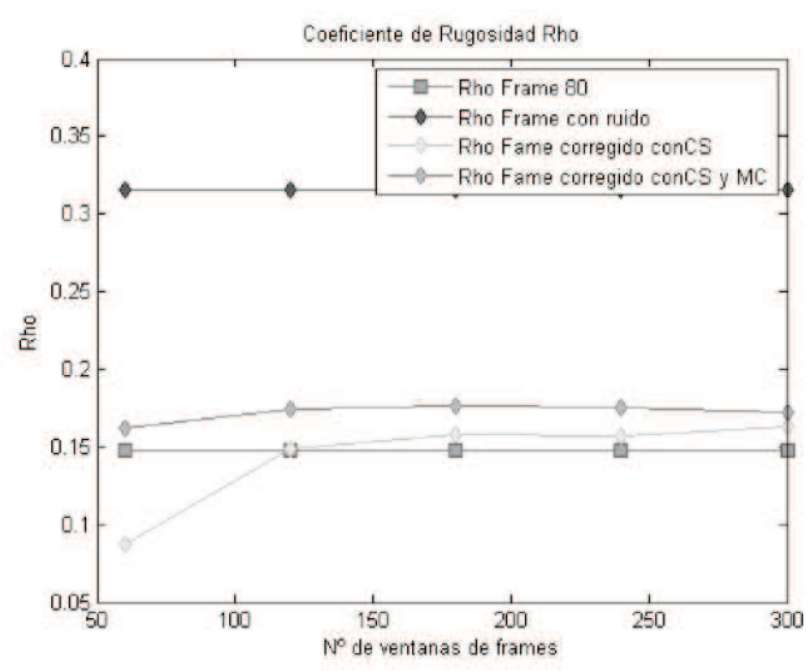

Figura 11. Coeficiente de rugosidad frame aleatorio, 1:30PM 


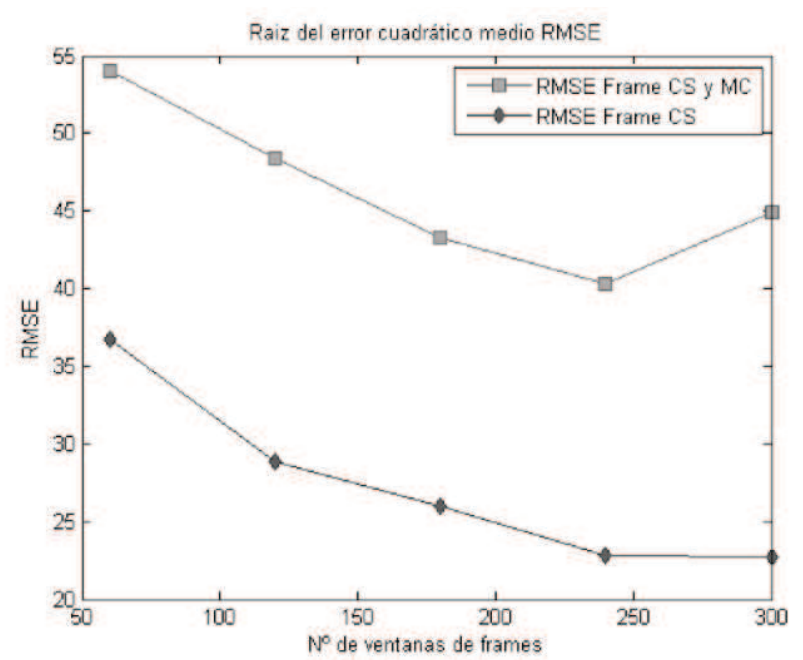

Figura12 . Figura de merito RMSE frame aleatorio 1:30PM

Al igual que en la técnica de rango constante, la técnica de estadísticas constantes presenta un buen desempeño en la corrección de no uniformidades en imágenes infrarrojas, en ambientes donde se presente una buena iluminación, este aspecto se ve reflejado en la Figura 10, la cual evidencia el buen desempeño en la eliminación del ruido espacial en la imagen, al aplicar las técnicas de CS y CS+MC.

La Figura 11 muestra que el coeficiente de rugosidad en las imágenes corregidas con CS y CS+MC es mucho más bajo que el de la imagen contaminada con ruido espacial; lo que implica que las técnicas de CS y CS+MC presentan un buen desempeño en la corrección de no uniformidades, ya que eliminan en gran parte el ruido espacial presente en las imágenes. Aunque el coeficiente de rugosidad de la imagen corregida con CS+MC sea mayor que al aplicar la técnica de CR, no implica que la corrección sea de bajo desempeño, como en el caso de la corrección con la técnica de $\mathrm{CR}+\mathrm{MC}$, ya que la técnica de maximización de contraste luego de corregir el ruido espacial, mejora el contraste en la imagen.

El RMSE de las imágenes corregidas con CS y CS+MC presenta un mayor error que en el caso de las técnicas de $C R$ y $C R+M C$, esto es debido a que la técnica de CS no supone una variabilidad de los pixeles en cada frame. Factor importante en la estimación de la radiación infrarroja, puesto que el valor de cada pixel en cada frame presenta una variación.
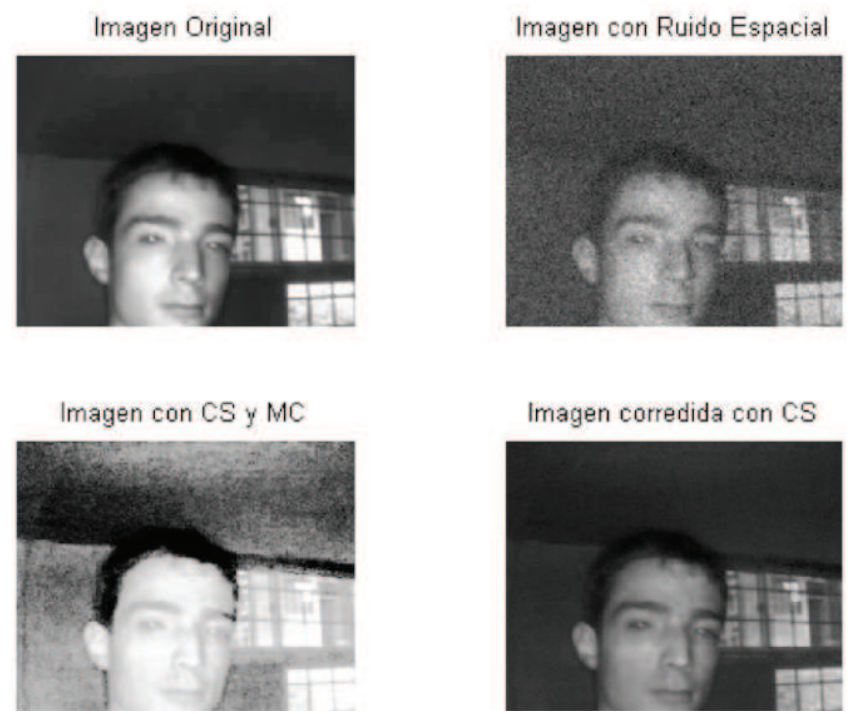

Figura 13. Corrección frame aleatorio, 5:45PM

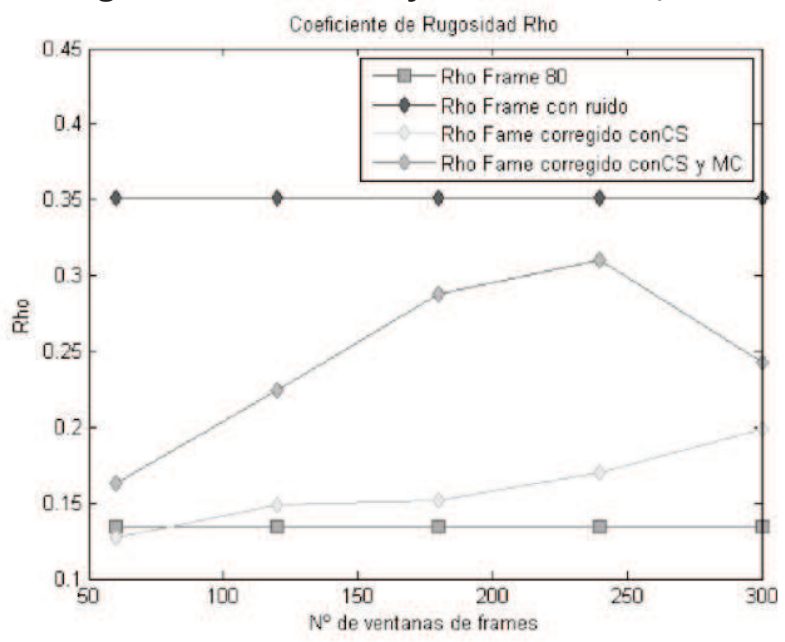

Figura 14 . Coeficiente de rugosidad frame aleatorio, 5:45PM

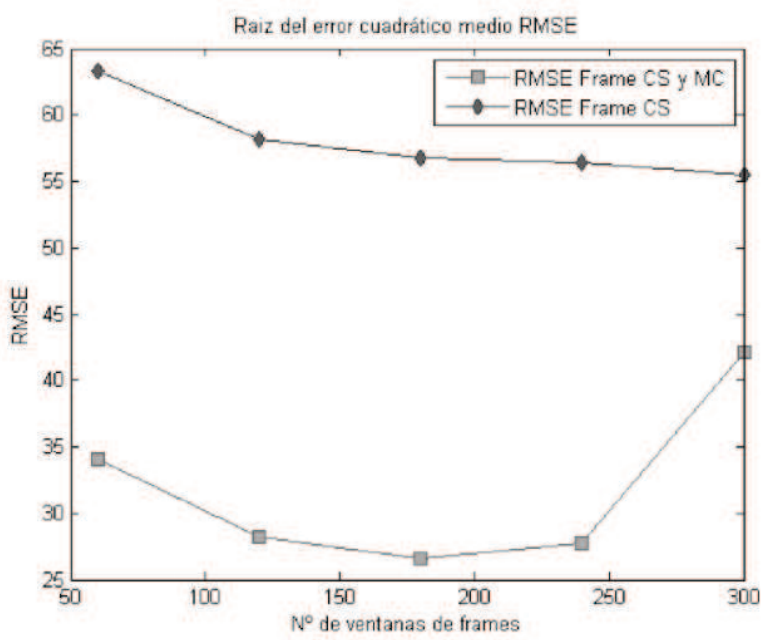

Figura . Figura de merito RMSE frame aleatorio 5:45PM 
En escenas de baja iluminación, como se muestra en la Figura 13, la estimación de la radiación infrarroja presenta un bajo desempeño utilizando las técnicas de CS y CS+MC, ya que la técnica de CS no considera la variabilidad de los pixeles en cada frame, esto ocasiona que en escenas de baja iluminación el ruido espacial presente en la imagen no sea eliminado completamente. Esta afirmación se puede evidenciar en las Figuras 14 y 15, en las cuales el coeficiente de rugosidad en las imágenes corregidas con CS y CS+MC es más alto que en la corrección de las imágenes con $C R$ y CR+MC, además el RMSE presenta un incremento ya que la técnica de CS no presenta una buena estimación de la radiación infrarroja presente en la escena.

\section{Contrastación de las técnicas estadísticas} constantes y rango constante en la corrección de no uniformidades

En la Figura 16 se muestra la corrección de un frame aleatorio adquirido a la 1:30PM hora en la cual la escena presenta una buena iluminación, contaminado con ruido de espacial del $35 \%$, utilizando las técnicas de $\mathrm{CS}+\mathrm{MC}$ y $\mathrm{CR}+\mathrm{MC}$.
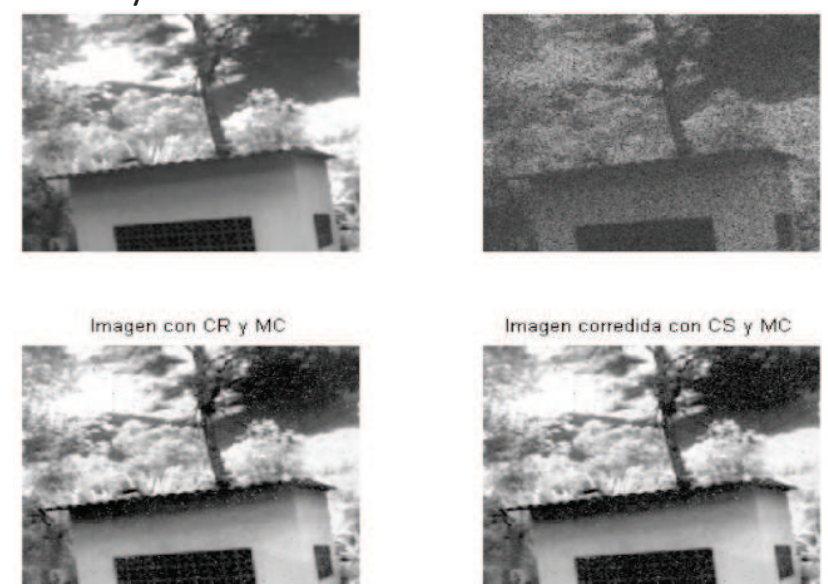

Figura 16. Corrección frame aleatorio, 1:30PM utilizando las técnicas de $\mathrm{CS}+\mathrm{MC}$ y $\mathrm{CR}+\mathrm{MC}$

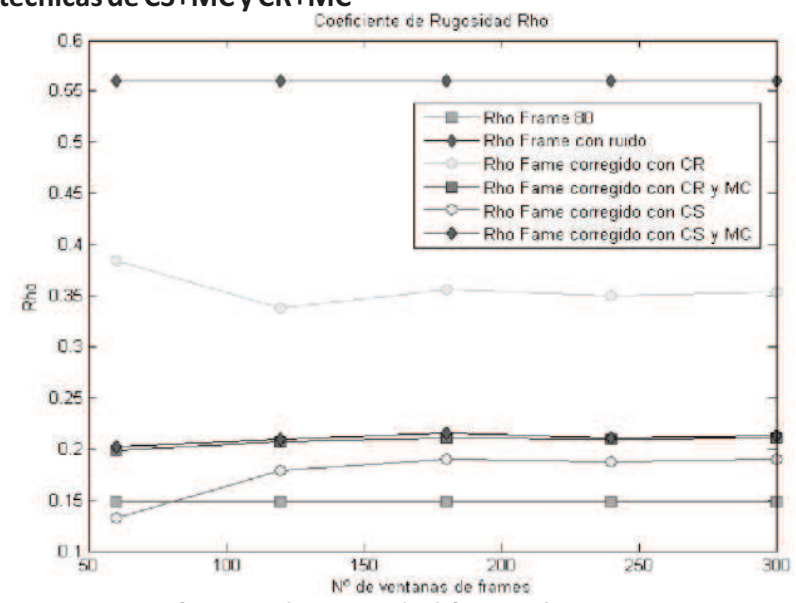

Figura 17. Coeficiente de rugosidad frame aleatorio 1:30PM

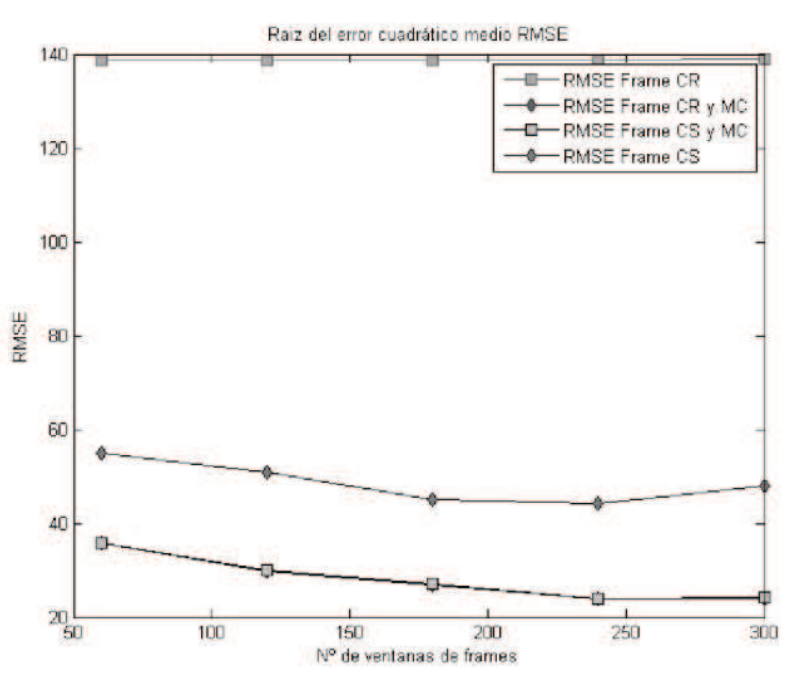

Figura . Figura de merito RMSE frame aleatorio 1:30PM

Al observar la Figura 16 se evidencia que las técnicas de $\mathrm{CS}+\mathrm{MC}$ y $\mathrm{CR}+\mathrm{MC}$, presentan un buen desempeño en la eliminación del ruido espacial presente en la imagen, resaltando que porcentaje de ruido con el que se contamino la imagen era alto. En el caso de la corrección con CS en la Figura 17 se observa claramente que es la técnica que presenta menor coeficiente de rugosidad lo cual no implica que sea la mejor técnica, además esta técnica condiciona el problema a escenas donde la iluminación sea muy buena y el porcentaje de ruido en la imagen sea bajo. Por ello al analizar la Figura 18 , se nota claramente que la técnica que mejor desempeño presenta debido a que su RMSE es el más bajo es la técnica de CR+MC ya que al suponer la variabilidad de los pixeles en cada frame, se mejora la estimación de la radiación infrarroja, la cual ocasionara un mejor desempeño en la corrección de no uniformidades en la imagen.

\section{DISCUSIÓN}

El algoritmo del Rango Constante, corrige efectivamente el ruido espacial y temporal, sin embargo presenta una disminución en su rendimiento cuando la radiación infrarroja es escasa, igualmente cuando los objetos de la escena se mantienen estacionarios por mucho tiempo ya que las varianzas locales son nulas lo que indetermina la estimación de la radiación infrarroja. Por otra parte el algoritmo de las estadísticas constantes hace una buena corrección del ruido espacial, sin embargo su rendimiento es más bajo 
comparada con la técnica del rango constante igualmente los rendimientos de las dos técnicas mejoran al efectuarse la maximización de contraste y compensación de iluminación.

Las técnicas del rango constante y estadísticas constantes no obtiene buenos rendimientos en ambientes con muy baja iluminación (oscuros), ya que el fondo de la imagen tiende a presentarse homogéneo, es decir con poca variación lo que causa indeterminaciones en la estimación de la radiación infrarroja.

En los casos en que la radiación infrarroja estimada es alta la técnica de maximización de contraste y compensación de iluminación, presenta saturaciones en la imagen de salida por lo tanto esta técnica debe utilizarse en escenas de baja iluminación siendo uno de los trabajos futuros mejorar la respuesta del algoritmo ante condiciones de iluminación altas.

\section{BIBLIOGRAFÍA}

1. Daniel Aquilino González Fernández “Contribuciones a las técnicas no destructivas para evaluación y prueba de procesos y materiales basadas en radiaciones infrarrojas" tesis doctoral, pp.72-80 2006.

2. P. M. Narendra, "Reference-free non-uniformity compensation for infrared imaging arrays," Proc. SPIE, Vol. 252, pp. 10-17, 1980.

3. D. Scribner, M. Kruer and J. Killiany, "Infrared Focal Plane Array Technology," Proceedings of the IEEE, Invited paper, Vol. 79 No.1, pp. 66-85, 1991.

4. Hernán Benitez, H. Loaiza, B. Vacca and J. Acevedo " Sistema de Visión Artificial para el Procesamiento y Análisis de Imágenes Infrarrojas",2006.

5. P. M. Narendra and N.A. Foss, "Shutterless fixed pattern noise correction for infrared imaging arrays," Proc. SPIE, Vol. 282, pp. 44-51, 1981.

6. B.K. Horn, “ Robot Vision” MIT Press,1996.

7. P. Belhumeur, J. Hespanha and D. Kriegman, “Eigenfaces vs Fisherfaces: Recognition using class specific Linear projection", IEEE Transactions on Pattern Analysis and Machine Intelligence, vol. 19, Issue 7, 1997.

8. A.S Georghiades, P.N. Belhumeur and D.J Kriegman, "From few to many: Illumination cone Models for Face Recognition Under Lighting and Pose”, , IEEE Trans. Pattern Analysis and Machine Intelligence, vol. 23, no. 6, pp. 630-660, 2001.

9. R. Basri and D. Jacobs, "Lambertian Reflectance and lineal Subspaces" IEEE International Conference on Computer Vision, 2001.

10. A.K. Jain, "Fundamentals of digital images processing". Prentice Hall International.

11. Short, J. Kittler and K. Messer "A Comparison of Photometric Normalization Algorithms for Face Verification" IEEE Int. Conf. Automatic Face and Gesture Recognition, 2004.

12. Edgar A. Rivas Araiza, Jorge D. Mendiola Santibañez, Gilberto Herrera Ruiz, Carlos A. González Gutiérrez, Mario Trejo Pereay G. J. Ríos Moreno “Contrast Enhancement and Illumination Changes Compensation”, 2007.

13. Rodrigo Andrés Reeves Díaz, “Compensación de ruido espacial en sistemas de imágenes con sensores infrarrojos" concepción chile 2002.

14. J. G Harris and Y. M. Chiang, "Continuous-Time calibration of VLSI sensors for gain and offset variations," Proc. SPIE, vol. 2474, pp. 23-33, 1995. 